\title{
Planificación de un centro de información territorial: la información como servicio a la sociedad en la toma de decisiones estratégicas
}

\author{
Planning a territorial information centre: information as a service to society
}

for strategic decision-making

\section{Concepción TorRes Liarte (1) y Ana Isabel Pallarés Ramo (2)}

(1) Consejo Económico y Social de Aragón (CESA), Avda. César Augusto 30-1 H, 50004 Zaragoza, España, ctorresl@aragon.es. (2) Centro de Documentación e Información Territorial de Aragón (CDITA), Edificio Puerta Cinegia, Coso 33-35-2 planta, 50003 Zaragoza, España, aipallares@aragon.es

\begin{abstract}
Resumen
En el nuevo contexto de la sociedad del conocimiento, un objetivo fundamental para la Ordenación Territorial es establecer los medios para disponer de la información que posibilite la adopción de decisiones relativas a la planificación del desarrollo. El acceso a este tipo de información es un instrumento necesario para las instituciones públicas y privadas, y para facilitar la toma de decisiones de los agentes económicos y sociales. En el presente artículo se ofrece un estudio relativo a la planificación del Centro de Documentación e Información Territorial de Aragón (CDITA), perteneciente a la Dirección General de Ordenación del Territorio del Gobierno de Aragón. Se presenta su resumen ejecutivo, visión, misión y objetivos estratégicos y operacionales, así como su análisis DAFO. A continuación se detallan las acciones realizadas por el CDITA para cumplir su objetivo de difundir la información territorial, principalmente la inclusión de sus contenidos en el Portal de Internet en el Gobierno de Aragón. Por último, se exponen los retos y perspectivas de futuro.
\end{abstract}

Palabras clave: Documentación territorial. Planificación. Centros de documentación. Administración pública. Internet. Portales institucionales. Aragón.

\section{Introducción}

El Centro de Documentación e Información Territorial de Aragón, creado por Decreto 162/2000, de 26 de julio del Gobierno de Aragón, adscrito a la Consejería de Política Territorial, Justicia e Interior, del Gobierno de Aragón, tiene la función de difundir la información y documentación territorial, de manera que permita su mejor conocimiento y facilite la toma de decisiones de los poderes públicos y de los agentes sociales y económicos. Es por ello que en los últimos años se ha apostado fuertemente por las nuevas tecnologías de la información y

\begin{abstract}
In the new context of the knowledge society, an essential aim for spatial planning is to establish the means to ensure the availability of the information needed to make decisions as far as development planning is concerned. Access to this type of information is a necessary tool for both public and private institutions, as well as for economic and social agents, to make decisions more easily. This paper offers a study related to the planning of the Centro de Documentación e Información Territorial de Aragón (CDITA) (Territorial Documentation and Information Centre of Aragón), which belongs to the Spatial Planning Section of Aragón's Regional Government. It presents its executive summary, vision, mission, strategic and operational objectives and SWOT analysis. Subsequently, this paper details the actions carried out by the CDITA to achieve its objective of diffusing territorial information, paying special attention to the publication of its contents on the Aragón's Regional Government Official Web Portal. Finally, future challenges and perspectives will be put forth.
\end{abstract}

Keywords: Territorial documentation. Documentation centres. Planning. Public administration. Internet. Institutional portals. Aragon (Spain).

la comunicación, ya que éstas son una herramienta fundamental para facilitar el intercambio y la difusión de la información entre los responsables de la Ordenación Territorial y de éstos con la sociedad.

Podemos citar dos ejemplos claros de esta apuesta por las nuevas tecnologías de la información. El primero es la presentación en mayo de 2007 del Sistema de Información Territorial de Aragón (SITAR), potente herramienta que da acceso a la cartografía y datos geográficos de Aragón y que tiene tres finalidades principales: facilitar información geográfica a aquellas uni- 
dades administrativas del Gobierno de Aragón que necesitan de la misma para desarrollar sus trabajos, difundir información geográfica pública de la comunidad autónoma para cualquier usuario de Internet, y facilitar la descarga de cartografía en múltiples formatos.

El segundo, en el que nos centraremos, es la inclusión en el Portal del Gobierno de Aragón en Internet de los contenidos del Centro de Documentación e Información Territorial de Aragón, dando así respuesta a las necesidades de información de la sociedad y avanzando en la consecución del objetivo de actuar como difusor de la información territorial.

Pero para llegar a ello iniciamos primero un análisis fundamental previo al plan estratégico que era necesario en dicho Centro de Documentación e Información Territorial de Aragón.

\section{Resumen ejecutivo}

Ante los nuevos retos relacionados anteriormente era necesaria una planificación estratégica. Para ello planteamos la visión y la misión que constituyen nuestro principal objetivo. Posteriormente realizamos un análisis DAFO de los entornos interno y externo del Centro que nos ayudaron a establecer los objetivos estratégicos y su concreción en los correspondientes objetivos operacionales. Finalmente, se analizan los recursos y acciones realizadas para plasmar esos objetivos.

\section{Visión y misión}

La visión consiste en ser el centro de referencia puntero relativo a temas territoriales en la Comunidad de Aragón.

Su misión es situar en unos niveles de innovación y modernidad los estudios territoriales y la producción cartográfica que hoy se hace en Aragón; así como facilitar y promover el acceso a esta información.

\section{Análisis DAFO}

El análisis DAFO del Centro de Documentación e Información Territorial de Aragón (CDITA) se presenta en la tabla I.

\section{Objetivos estratégicos y operacionales}

Los objetivos estratégicos y operacionales se detallan en la tabla II, en la página siguiente.
ANALISIS DAFO CENTRO DE DOCUMENTACIÓN E INFORMACIÓN TERRITORIAL (CDITA)

\begin{tabular}{|c|c|c|}
\hline & AMENAZAS & OPORTUNIDADES \\
\hline & $\begin{array}{l}\text { - Alta dependencia de los } \\
\text { cambios políticos y } \\
\text { desoondinación entre los } \\
\text { diferentes Departamentos. } \\
\\
\text { - Escasa formación tecnológica } \\
\text { de algunos de los usuarios. } \\
\text { Rechazo a la tecnología } \\
\text { - Desonnocimiento del Centro } \\
\text { por parte de otros } \\
\text { Departamentos, asi como de } \\
\text { sus proyectos. }\end{array}$ & $\begin{array}{l}\text { - Apoyo institucional } \\
\text { - Reconocimiento de nuestras } \\
\text { capacidades por parte de la } \\
\text { propia institución (Gobiemo) } \\
\text { - Nuevo local (escaso si se } \\
\text { amplia la oferta de productos y } \\
\text { servicios) } \\
\text { - Impacto de las nuevas } \\
\text { tecnologías en el ámbito } \\
\text { cartográfico y de } \\
\text { documentación. } \\
\text { - Vocación de servicio a la } \\
\text { sociedad del Centro. } \\
\text { - Posibilidad de integración de } \\
\text { diversos servicios: cartografia } \\
\text { propiamente dicha y } \\
\text { documentación territorial. } \\
\text { - Dirección clara de la Unión } \\
\text { Europea y las instituciones } \\
\text { cartográficas y documentales } \\
\text { hacia la Sociedad de la } \\
\text { Información y del } \\
\text { Conocimiento. }\end{array}$ \\
\hline FORTALEZAS & Estrategias defen sivas & Estrategias ofensivas \\
\hline $\begin{array}{l}\text { - Encuadrado en una } \\
\text { sólida Institución: } \\
\text { Gobierno de Aragón } \\
\text { - Maneja información } \\
\text { estratágica junto a una } \\
\text { de carácter divulgativo } \\
\text { (Cartografía urbana y } \\
\text { rústica de Aragón. } \\
\text { Estudios de carácter } \\
\text { estratégios. Elaboración } \\
\text { de mapas de carácter } \\
\text { divulgativo) } \\
\text { - Personal cualificado en } \\
\text { usos de software } \\
\text { indicado. } \\
\text { - Capacidad en la gestión } \\
\text { de recursos actuales } \\
\text { - Rapidez en la atención } \\
\text { - Dependencia de sus } \\
\text { usuarios: el servicio es } \\
\text { casi exclusivo } \\
\text { - Presupuesto suficiente si } \\
\text { se justifica } \\
\text { debidamente. } \\
\text { - Buenos sistemas de } \\
\text { información y acoeso a } \\
\text { las nuevas tecnologías } \\
\text { Buena sintonía oon los } \\
\text { centros nacionales } \\
\text { hermanos (catastro, } \\
\text { CNIG, etc) }\end{array}$ & $\begin{array}{l}\text { - Productos y servicios que } \\
\text { atiendan a la demanda } \\
\text { incipiente: } \\
\\
\\
\text { v Finalización de la cartografía } \\
\text { completa de Aragón. } \\
\checkmark \text { Nuevas ortofotos } \\
\checkmark \text { Completar la oolección de } \\
\text { libros "Territorio" editados } \\
\text { por nosotros de las comarcas } \\
\checkmark \text { Dossieres. } \\
\checkmark \text { Informes relativos a } \\
\text { directrices territoriales y } \\
\text { otros. } \\
\text { - Plan tecnológico para } \\
\text { actualizar nuestros recursos }\end{array}$ & $\begin{array}{l}\text { valor añadido. Contratación } \\
\text { de estudios externos (en } \\
\text { colaboración con el Servicio } \\
\text { de Estratagias Territoriales). } \\
\text { El Centro oomo editor de } \\
\text { contenidos. } \\
\text { - Sistema de racionalización de } \\
\text { Documentación Territorial } \\
\text { existente en todos los } \\
\text { Departamentos del Gobierno. } \\
\text { Inventario topológioo de } \\
\text { todos los estudios y } \\
\text { documentos referentes al } \\
\text { territorio. } \\
\text { - Plan de formación tecnológica } \\
\text { de usuarios. } \\
\text { - Marketing de los productos } \\
\text { del CDITA entre los usuarios. } \\
\text { - Posibilidad de crear } \\
\text { comunidades virtuales u otra } \\
\text { tipología para grupos } \\
\text { especilizados. }\end{array}$ \\
\hline DEBILIDADES & Estrategia de supervivencia & Estrategia de reorientación \\
\hline $\begin{array}{l}\text { - La toma de decisiones } \\
\text { reside únicamente en la } \\
\text { alta dirección. } \\
\text { - Al formar parte del } \\
\text { gobiemo, hay una gran } \\
\text { dependencia un su } \\
\text { planificación estratégica } \\
\text { de las decisiones politicas, } \\
\text { al menos a oorto plazo. } \\
\text { - Escaso oonocimiento del } \\
\text { Centro en la sociedad } \\
\text { aragonesa, así como de } \\
\text { los servicios que ofreoe } \\
\text { - Mala difusión de los } \\
\text { reaursos } \\
\text { - Inexistencia de } \\
\text { información actualizada } \\
\text { de estudios actuales del } \\
\text { ternitorio. Obsolesoencia } \\
\text { de los existentes. } \\
\text { - Escasez de reaursos } \\
\text { humanos para la labor } \\
\text { que es neoesario realizar. } \\
\text { Lastre del trabajo diario. }\end{array}$ & \begin{tabular}{|l|} 
- Continuar oon los productos \\
que ofreoemos, si es posible \\
con un mayor valor añadido. \\
- Considerar el Centro como \\
centro de referencia en la \\
región de los temas de \\
cartografía y ordenación del \\
teritorio. \\
- Plan de Formación permanente \\
para el personal en plantilla. \\
- Nuevas contrataciones de \\
personal. Desarrollar una \\
política de reaursos humanos \\
que defina las nuevas \\
funciones y perfiles \\
profesionales basados en \\
capacidades y neoesidades del \\
servicio.
\end{tabular} & $\begin{array}{l}\text { - Es neoesario dar a conocer a la } \\
\text { sociedad en general así como a } \\
\text { los técnicos la existencia del } \\
\text { Centro ocmor referencia central } \\
\text { de la región. } \\
\text { - Establecer nuevos canales de } \\
\text { comunicación } \\
\text { - Crear grupos de trabajo } \\
\text { (extemoso intemos) para crear } \\
\text { y desarrollar proyectos } \\
\text { directamente relacionados oon el } \\
\text { Centro. Posiblemente en relación } \\
\text { directa oon el resto de la } \\
\text { Dirección General. } \\
\text { - Crear una estrategia de } \\
\text { planificación para adelantarse a } \\
\text { los cambios, a largo plazo, o a } \\
\text { menos a nivel de legislatura (4 } \\
\text { años) }\end{array}$ \\
\hline
\end{tabular}

Tabla I. Análisis DAFO. 
OBJETIVOS ESTRATÉGICOS Y OPERACIONALES

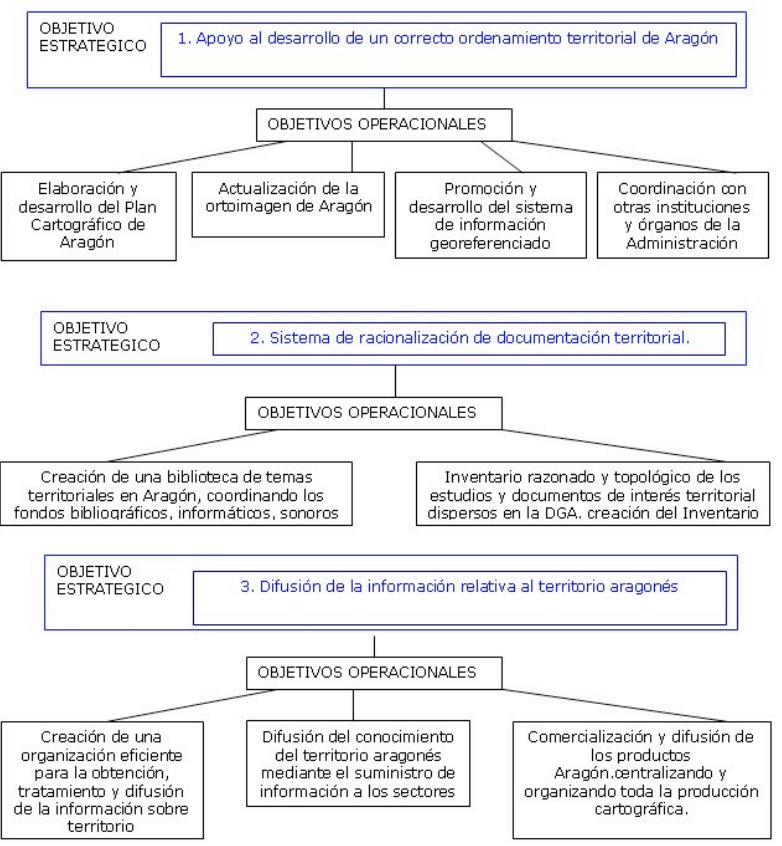

Tabla II. Objetivos estratégicos y operacionales.

\section{Resultado final}

Uno de los resultados de la planificación descrita con anterioridad es la incorporación, en noviembre de 2007, de los contenidos desarrollados por el CDITA en el Portal del Gobierno de Aragón en Internet. A continuación se describen dichos contenidos.

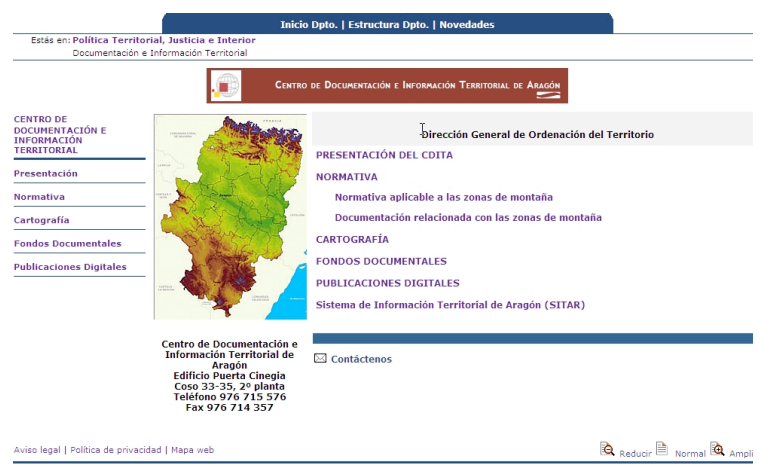

Figura 1. Página principal del Centro de Documentación e Información Territorial de Aragón.

\subsection{Acceso}

Se accede al CDITA desde la página principal del Gobierno de Aragón, www.aragon.es, de dos formas distintas: a) dentro de la sección Departamentos y Organismos Públicos, en el Departamento de Política Territorial, Justicia e Interior, y dentro de éste, en el apartado Información Territorial; y b) dentro de la sección Índi- ce Temático, en el apartado Información Territorial.

\subsection{Secciones}

\subsubsection{Presentación del CDITA}

Consiste en una descripción del centro, ubicación y horarios de atención al público, servicios que ofrece y marco legal que regula su funcionamiento. (Figura 2).

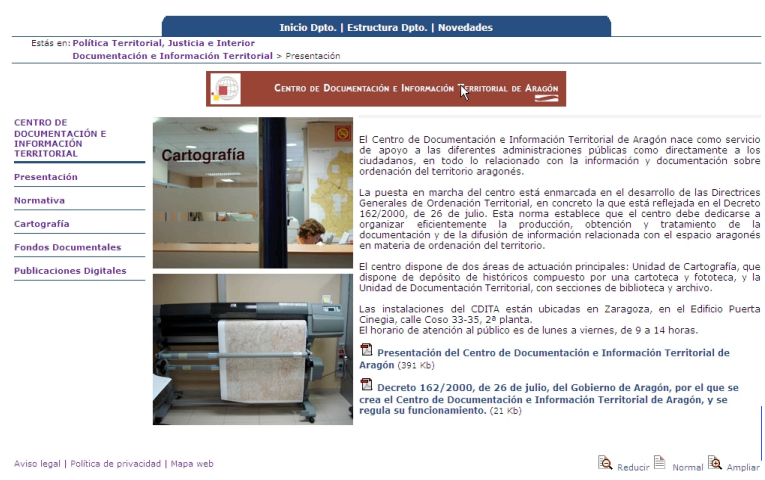

Figura 2. Presentación del Centro de Documentación e Información Territorial de Aragón.

\subsubsection{Normativa}

Incluye una relación de normativa sobre ordenación territorial, dividida en varios apartados:

- Normativa sobre ordenación territorial, de la Comunidad Autónoma de Aragón y la Unión Europea, vigente y en proceso de tramitación.

- Normativa sectorial con incidencia en la ordenación territorial, de la Comunidad Autónoma de Aragón y estatal, dividida en varios apartados: urbanismo, medio ambiente, organización administrativa, infraestructuras de transporte y movilidad, patrimonio cultural, agricultura, industria, turismo, ciencia y tecnología, salud y demografía.

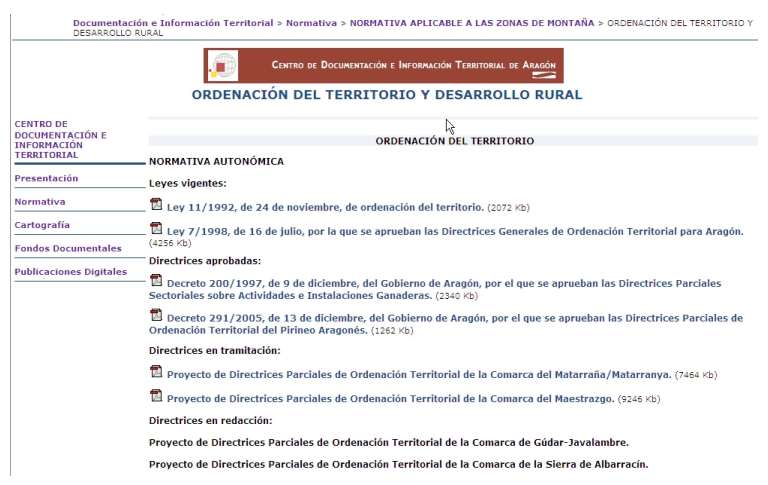

Figura 3. Normativa. 
- Normativa aplicable a las zonas de montaña, dividida en varios apartados: ordenación del territorio y desarrollo rural, medio ambiente, urbanismo y otra normativa sectorial.

- Documentación relacionada con las zonas de montaña, que incluye otro tipo de documentos como recomendaciones, convenios, declaraciones, dictámenes o actas de congresos. Se ha dividido en tres epígrafes: ordenación del territorio y desarrollo rural, medio ambiente y otros documentos sectoriales.

\subsubsection{Cartografía}

Contiene una descripción de la cartografía disponible en el Centro, una relación de los distintos servicios cartográficos que pueden solicitarse y un enlace directo al Sistema de Información Territorial de Aragón (SITAR) (figuras 4 y 5).

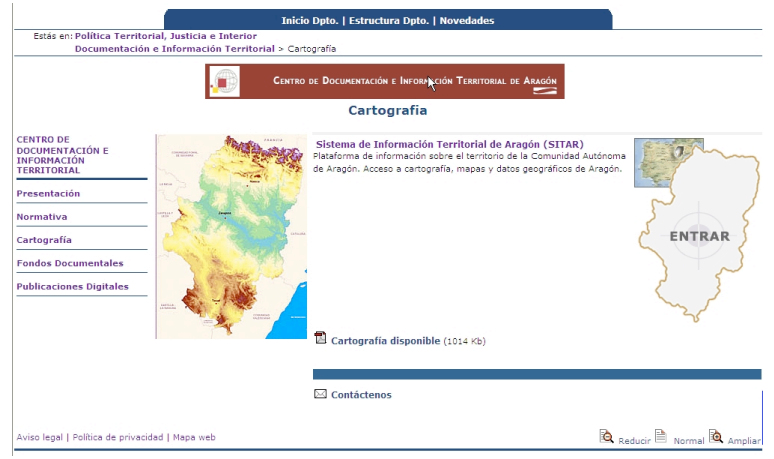

Figura 4. Cartografía.

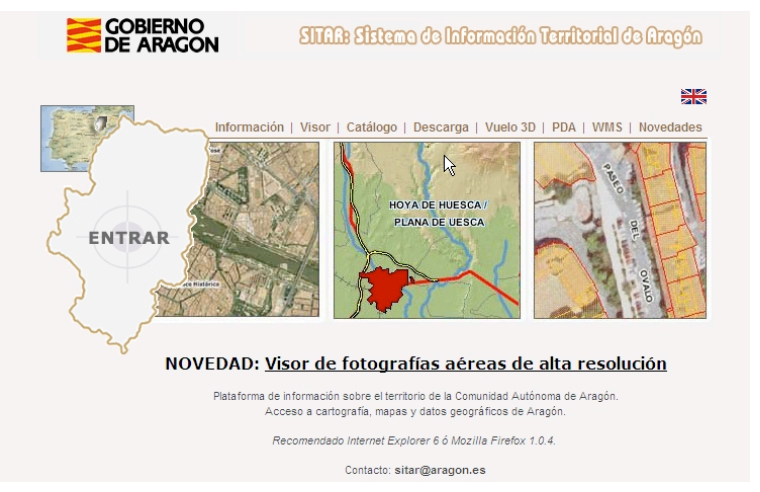

Figura 5. Página principal del Sistema de Información Territorial de Aragón (SITAR).

\subsubsection{Fondos documentales}

Se accede la base de datos de los fondos documentales del CDITA, de aproximadamente 20.000 registros. Los fondos documentales se han estructurado en los apartados:

- Hemeroteca, con publicaciones periódicas especializadas en temas relacionados con la ordenación del territorio, en español y otros idiomas.

- Biblioteca, con monografías especializadas en temas territoriales.

- Bibliografías, con referencias a libros publicados en España sobre ordenación territorial.

Mediante un formulario de consulta se puede acceder a las distintas referencias bibliográficas. En la actualidad esta base de datos bibliográfica se encuentra en proceso de actualización.

También se accede desde aquí a la Guía de recursos cartográficos, documentales y de internet del Centro de Documentación e Información Territorial que se realizó en el año 2005 (figura $6)$.

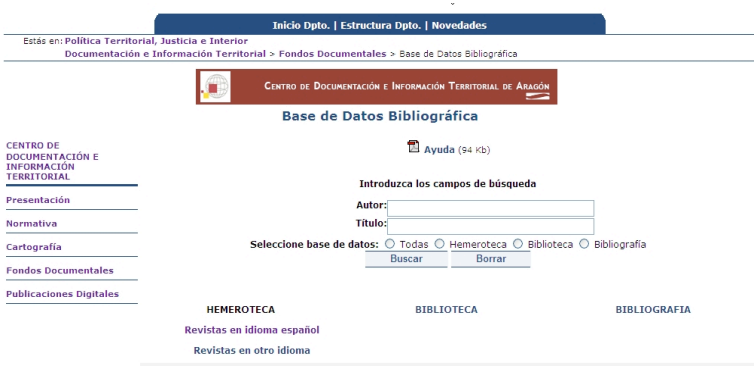

Figura 6. Base de datos bibliográfica.

\subsubsection{Publicaciones digitales}

Se incluyen las publicaciones que se realizan desde el CDITA, en formato PDF:

- La revista Territorio, Comarcas de Aragón, y la revista Territorio y Desarrollo Local, continuación de la anterior, son revistas muertas y desde aquí se pueden consultar todos sus números.

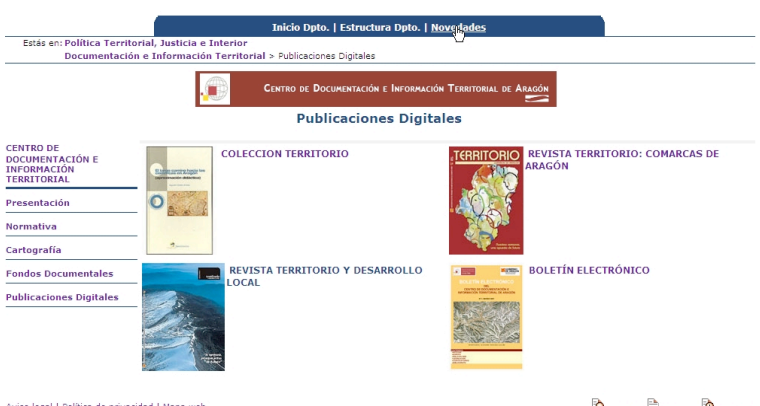

Figura 7. Publicaciones digitales.

- La Colección Comarcas es el resultado del proceso de comarcalización de Aragón, y tiene el objetivo de dar a conocer las distintas comarcas de la Comunidad Autónoma al ciudadano en general. Cada volumen de la co- 
lección se centra en una comarca, y el usuario puede descargarse la publicación entera o el capítulo que sea de su interés.

- El Boletín Electrónico del Centro de Documentación e Información Territorial de Aragón, de periodicidad mensual, incluye información de actualidad referente a la ordenación territorial, y consta de varias secciones: Noticias, Legislación, Europa, Enlaces Web y Bibliografía (figura 7 ).

\section{Perspectivas}

El volumen de información territorial disponible en Internet, como en cualquier materia, ha crecido exponencialmente en los últimos años. En este contexto de un elevado volumen de información, resultan claves los sitios web que aglutinen y organicen el acceso a la información que sea relevante y de calidad. Para ello, se hará un seguimiento y evaluacion permanente que redundará en la mejora continua del servicio.

En esta línea, el objetivo del Centro de Documentación e Información Territorial de Aragón es seguir ofreciendo una información actualizada en la red, con la revisión constante del Sistema de Información Territorial de Aragón, la actualización periódica de la normativa, la publicación del Boletín Electrónico, y la perspectiva de incluir nuevas secciones en la web, como un directorio de direcciones de internet relativas a la ordenación territorial.

\section{Bibliografía}

Asociación Española de Normalización y Certificación (1999). Documentación. 3. ${ }^{a}$ ed. Madrid: AENOR, 1999.

Birdsall, D. (1994). A new strategic planning model for academic libraries. // College and Research Libraries. (1994) 149-159.

Butler, M. M.; Davis, H. (1992). Strategic planning as a Catalyst for change in the 1990s. // College and Research Libraries. (1992) 393-403.

González Uceda, L. (2007). Información y documentación para las administraciones públicas. Madrid: Madrid (Comunidad Autónoma), Publicaciones, 2007.

Newsome, James; Mclnerney, Claire (1990). Evironmental Scanning and the Information Manager. // Special Libraries. 4:81 (1990) 285-293).

Ramos Simón, L. F. (1995). Dirección, administración y marketing de empresas e instituciones documentales. Madrid: Síntesis, 1995.

Tom Whitehall (1992). Calidad en los servicios bibliotecarios y de la información: un informe. // Library Management. $13: 5$ (1992) 23-35. 